\title{
UMAP 2017 THUM Workshop Chairs' Welcome
}

The importance of user modeling and personalization is taken for granted in several scenarios. According to this widespread paradigm, each user can be modeled through some (explicitly or implicitly gathered) information about her knowledge or about her preferences, in order to adapt the behavior of a generic intelligent system to her specific characteristics. However, the recent spread of social network and self-tracking devices has totally changed the rules for personalization. On one side, the spread of social network platforms radically changed and renewed many consolidated behavioral paradigms.

Thanks to the heterogeneous nature of the discussions that take place on social networks, a lot of new data are continuously available and can be gathered and exploited to build richer and more complete user models, to discover latent communities, to infer information about users' emotions and personality traits, and also to study very complex phenomena, such as those related to the psycho-social sphere, in a totally new way. At the same time, self-tracking devices are becoming more and more pervasive, and a plethora of personal data is today available by exploiting such tools.

These devices model and track a lot of signals that pure content-based information which is commonly spread on social networks can't actually handle. Reasoning on these data can enable predictions about the user's behavior, health, and goals. As a consequence, it is very important to think about a new generation of user models that are able to effectively merge the information coming from both information sources, while also taking into account the fact that user models evolve over time.

The workshop aims at bringing together researchers and practitioners working in order to look at the challenges from the point of view of the temporal and holistic aspects in user modelling. We accepted five contributions to be discussed during the workshop.

- HappyFit: Time-aware Visualization for Daily Physical Activity and Virtual Reality Games

- An Approach to Improve Physical Activity by Generating Induvial Implementation Intentions

- User's Social Media Profile as Predictor of Empathy

- Social Recommendation with Time and Sentiment Analysis

- Predicting Age and Gender by Keystroke Dynamics and Mouse Patterns

The main goal of the workshop is to stimulate the discussion around problems, challenges and research directions regarding the exploitation of content-based information sources (Big, Social and Linked Data) along with Personal Information sources (gathered through personal devices) for personalization and adaptation task and to foster the design of a new generation of intelligent usercentered systems.

$\begin{array}{llll}\text { Cataldo Musto } & \text { Amon Rapp } & \text { Veronika Bogina } & \text { Federica Cena } \\ \text { University of Bari } & \text { Università di Torino } & \text { University of Haifa } & \text { Università di Torino } \\ \text { Frank Hopfgartner } & \text { Judy Kay } & \text { David Konopnicki } & \text { Tsvi Kuflik } \\ \text { University of Glasgow } & \text { University of Sydney } & \text { IBM Research } & \text { University of Haifa } \\ \text { Bamshad Mobasher } & \text { Giovanni Semeraro } & \text { Workshop Chairs } & \\ \text { DePaul University } & \text { University of Bari } & & \end{array}$




\title{
THUM Workshop Organization
}

Organizing Committee: Cataldo Musto (University of Bari, Italy)

Amon Rapp (Università di Torino, Italy)

Veronika Bogina (The University of Haifa, Israel)

Federica Cena (Università di Torino, Italy)

Frank Hopfgartner (University of Glasgow, UK)

Judy Kay (The University of Sydney, Australia)

David Konopnicki (IBM Research, Haifa)

Tsvi Kuflik (The University of Haifa, Israel)

Bamshad Mobasher (DePaul University, Chicago, USA)

Giovanni Semeraro (University of Bari, Italy)

\author{
Program Committee: Liliana Ardissono (Università di Torino, Italy) \\ Shlomo Berkovsky (Data61 CSIRO, Australia) \\ Robin Burke (DePaul University, Chicago, USA) \\ Alfred Kobsa (University of California, USA) \\ Pasquale Lops (University of Bari “Aldo Moro”, Italy) \\ Inbal Ronen (IBM Haifa Labs, Israel) \\ Giuseppe Sansonetti (Università Degli Studi Roma Tre, Rome, Italy) \\ Amit Tiroshi (Atlassian, Australia) \\ Alejandro Bellogín (Universidad Autónoma de Madrid, Spain) \\ Ivan Cantador (Universidad Autónoma de Madrid, Spain) \\ Peter Dolog (Aalborg University, Denmark) \\ Cristina Gena (Università di Torino, Italy) \\ Fabio Gasparetti (Università degli Studi Roma Tre, Italy) \\ Bob Kummerfeld (University of Sydney Australia) \\ Fedelucio Narducci (University of Bari, Italy) \\ Marco Polignano (University of Bari, Italy) \\ Shaghayegh Sahebi (Univesity of Albany, United States) \\ Giuseppe Sansonetti (Università degli Studi Roma Tre) \\ Italy Christoph Trattner (Modul University, Vienna \\ Till Plumbaum (TU Berlin, Germany) \\ Markus Zanker (Free University of Bozen, Bolzano)
}

